\title{
Photosystem II - Mediated Cyclic Photophosphorylation
}

\author{
Charies F. Yocum \\ Dept. of Cell and Molecular Biology \\ The University of Michigan \\ Ann Arbor, Michigan 48104
}

\section{Received December 3,1975}

SUMMARY: DCMU-sensitive synthes is of ATP can be shown to continue in KCNtreated chloroplasts after cessation of $\mathrm{O}_{2}$ evolution. The catalyst for this reaction, p-phenylenediamine, also stimulates synthesis of ATP in $\mathrm{NH}_{2} \mathrm{OH}$-treated chloroplasts, but at much higher rates. This ATP synthesis can be observed in the presence of the quinone antagonist dibromothymoquinone, and under the appropriate conditions it is completely sensitive to DCMU. Since neither uptake nor evolution of $\mathrm{O}_{2}$ can be observed during illumination, these results are interpreted as evidence for catalys is of cyclic photophosphorylation by photosys tem II.

INTRODUCTION. The p-phenylenediamines are versatile mediators of electron flow in isolated chloroplasts. Under conditions where electron transport to photosystem I is interrupted by $K C N(1)$ or by the quinone antagonist dibromothymoquinone $(D B M I B)^{\top}(2)$, oxidized p-phenylenediamines can be shown to catalyze a DCMU-sensitive oxygen evolution which requires only photosystem II activity. This reaction is coupled to ATP synthesis, yielding approximately half the ATP (per electron pair) obtained when noncyclic electron transport from water to either ferricyanide or methyl viologen is assayed (3). In the presence of DCMU, ascorbate, and methyl viologen, the p-phenylenediamines also catalyze non-cyclic oxygen uptake and phosphorylation associated with photosystem I (4), while in the absence of ascorbate and methyl viologen, they catalyze a photosystem I (5) cyclic photophosphorylation reaction.

In the course of experiments to measure the stoichiometry of electron flow and ATP synthesis using KCN-treated chloroplasts and p-phenylenediamine plus ferricyanide as the electron acceptor system, it was noted that synthesis of ATP continued after cessation of $\mathrm{O}_{2}$ evolution. Further exploration of

\footnotetext{
'Abbreviations: DBMIB, dibromothymoquinone; PD, p-phenylenediamine; DCMU, 3-(3,4-dichloropheny 1)-1,1-dimethylurea.
} 
this observation has provided evidence, described in this communication, for photophosphorylation mediated by photosystem II cyclic electron flow.

MATERIALS AND METHODS. Isolated chloroplasts were prepared by homogenizing $100 \mathrm{gm}$ of spinach leaves in $250 \mathrm{ml}$ of cold Tricine buffer (20 mM, pH 8.0) containing sucrose $(0.4 \mathrm{M}), \mathrm{NaCl}(15 \mathrm{mM})$, and bovine serum albumin $(2 \mathrm{mg} / \mathrm{mT})$ for $15 \mathrm{sec}$. The resulting slurry was passed through four layers of cheesecloth and centrifuged $(1 \mathrm{~min})$ at $1,000 \times \mathrm{x}$. The supernatant from this step was centrifuged (10 min) at $5,000 \times g$, the pellet was resuspended in the homogenizing medium, and the centrifugation was repeated. The washed pellet was then resuspended in homogenizing medium, and the chlorophyll concentration was determined $(6)$. These chloroplasts were used for either $\mathrm{KCN}-$ or $\mathrm{NH}_{2} \mathrm{OH}-$ inhibition treatments $(1,7)$ and then stored in homogenizing medium at $-70^{\circ} \mathrm{C}$ until assay. p-Phenylenediamine (PD) was obtained from Eastman and recrystallized after charcoal treatment to remove impurities. The white crystals were dissolved in water to produce a $20 \mathrm{mM}$ stock solution. DBMIB was a kind gift of Dr. H.E. Good. A $2 \mathrm{mM}$ stock solution was prepared in ethanol for use in assays. ADP was obtained from Sigma, and all other chemicals were of the purest grade available.

When oxygen evolution or uptake was monitored, assays were done in a thermostatted cuvette $\left(25^{\circ} \mathrm{C}\right)$ fitted with a Clark electrode (YSI). The reaction mixture (1.6 $\mathrm{m} 1$ ) contained $20-40$ ug chlorophyl1, Tricine (50mM, pH 8.0), $\mathrm{NaCl}(60 \mathrm{mM}), \operatorname{ADP}(1.0 \mathrm{mM}), \mathrm{MgCl}_{2}(3.0 \mathrm{mM}), P D$ and ferricyanide ${ }_{6}$ at varying concentrations, and inorganic phosphate $(5.0 \mathrm{mM})$ plus $32 \mathrm{p}\left(10^{6}\right.$ $\mathrm{CPM}$ ). When cyclic photophosphorylation was measured the same reaction mixture was placed in $5 \mathrm{ml}$ test tubes for $i 17$ umination. Saturating white light $\left(10^{6}\right.$ ergs $\mathrm{cm}^{-2} \mathrm{sec}^{-1}$ ) was provided either by a microscope lamp or by photoflood lamps. Reactions were terminated after illumination by addition of $0.2 \mathrm{ml}$ of $30 \%$ TCA, and ATP synthes is was determined by gas flow counting after extraction of unreacted phosphate by Avron's procedure (8).

RESULTS. Figure 1 shows the time course of $\mathrm{O}_{2}$ evolution and ATP synthesis in $\mathrm{KCN}$-treated chloroplasts in the presence of PD $(250 \mu \mathrm{M})$ plus ferricyanide $(250 \mu M)$. Under these conditions of assay, $0_{2}$ evolution stops after approximately 1 min of illumination, but ATP synthesis continues for up to 5 min. Since $\mathrm{KCN}$-treated chloroplasts are unable to transfer electrons beyond cytochrome $f(9)$, the results in Figure 1 were interpreted to indicate catalysis of cyclic photophosphorylation by photosystem II. When ferricyanide was omitted from the reaction mixture, $\mathrm{O}_{2}$ evolution was no longer observed, but low rates of ATP synthes is (9-12 umoles/hr/mg chlorophy11) were still obtained and this reaction was completely inhibited in the presence of $10 \mu \mathrm{M}$ DCMU. These results showed that $P D$ was the catalyst of electron flow rather than ferricyanide, and strongly suggested that ATP synthesis in this assay system was arising from a photosystem II cyclic reaction. 


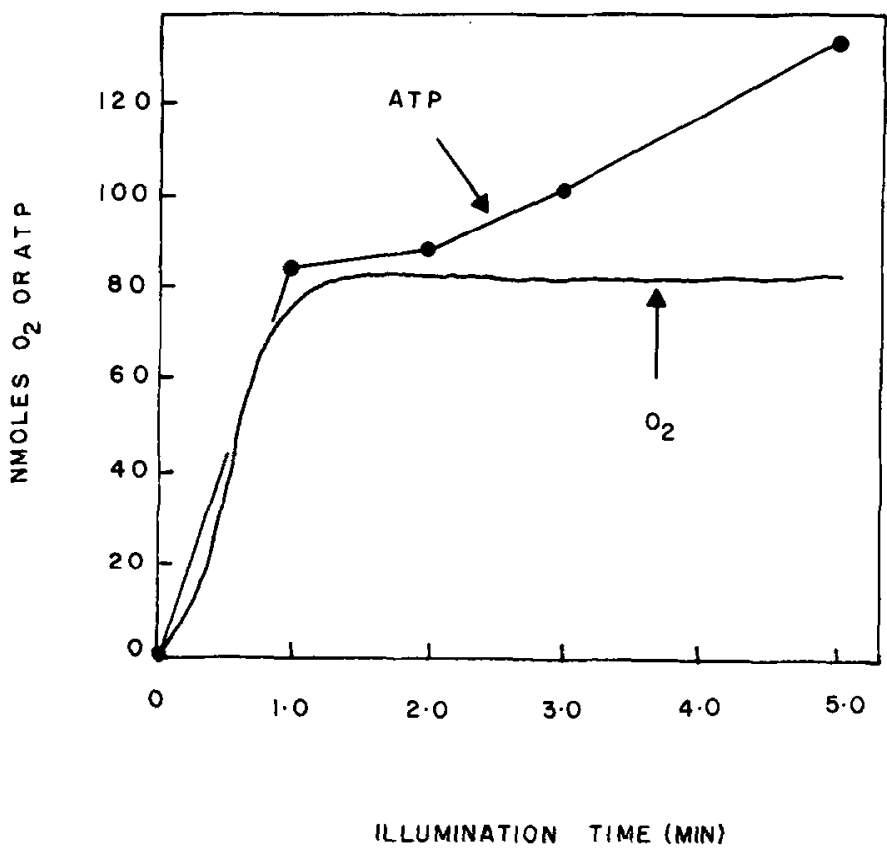

Figure 1. ATP Synthesis and $\mathrm{O}_{2}$ Evolution Catalyzed by $\mathrm{KCN}$-Treated Chloroplasts in the Presence of PD $(250 \mu \mathrm{M})$ plus Ferricyanide $(250 \mu \mathrm{M})$.

Attempts to improve the yield of ATP in $\mathrm{KCN}$-treated chloroplasts by increasing the concentration of $P D$ were without success, implying that some process in photosystem II was limiting the rate of cyclic electron transport or photophosphorylation. In order to see whether water photooxidation was limiting the cycle, photophosphorylation rates were determined using $\mathrm{NH}_{2} \mathrm{OH}-$ treated chloroplasts which cannot photooxidize water, and the results were compared to those obtained with $\mathrm{KCN}$-treated chloroplasts. Representative data from these experiments are shown in Table I. It is clear from these results that deletion of $\mathrm{O}_{2}$ evolution produced more efficient photophosphorylation when PD was the catalyst, but at the same time this reaction was not completely blocked by addition of DCMU, which indicated that under the conditions shown in Table I, photosystem I was contributing to electron flow and phosphorylation.

Since $\mathrm{NH}_{2} \mathrm{OH}$-treated chloroplasts gave higher rates of ATP synthesis 
Table I

ATP Synthesis in $\mathrm{KCN}$ - and $\mathrm{NH}_{2} \mathrm{OH}$-Treated Chioroplasts*

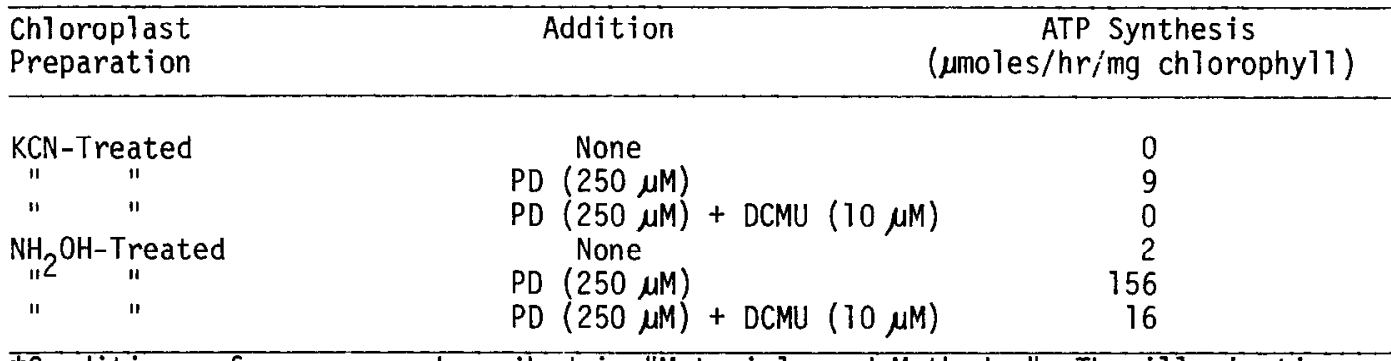

"Conditions of assay are described in "Materials and Methods." The illumination time was $1 \mathrm{~min}$.

with $\mathrm{PD}$ than did $\mathrm{KCN}$-treated preparations, it was of interest to see whether the photosystem I component of photophosphorylation could be eliminated from the $\mathrm{NH}_{2} \mathrm{OH}$-treated preparations to produce photosystem II cyclic activity. When the concentration of PD was decreased in the assay system, ATP synthesis became progressively more sensitive to DCMU, but a complete inhibition of activity could not be achieved. If, however, DBMIB (10 $\mu M)$ was added to assays along with concentrations of PD not exceeding $65 \mu \mathrm{M}$, conditions were created whereby ATP synthes is was completely sensitive to DCMU. Table II presents the results of these experiments along with data showing the rates of ATP synthesis both in the absence of and in the presence of high concentrations of PD. These results show that DBMIB alone does not appreciably stimulate ATP synthesis. The combination of $10 \mu \mathrm{M}$ DBMIB and 62.5 $\mu M$ PD, however, produced a photophosphorylation reaction which was completely inhibited by DCMU. A higher concentration of PD (1300 $\mu \mathrm{M})$, on the other hand, produced a rate of ATP synthesis which could not be completely inhibited by either DBMIB or DCMU alone, or when added together. This activity must therefore involve a substantial contribution from photosystem I.

It has been shown that DBMIB is oxidized by oxygen (10), and it is thus possible that the activities shown in Table II were a product of non-cyclic electron flow to $\mathrm{O}_{2}$ via DBMIB. This consideration was eliminated by experi- 
Table II

Effect of DBMIB and DCMU on PD-Catalyzed ATP Synthesis in $\mathrm{NH}_{2} \mathrm{OH}$-Treated Chloroplasts *

\begin{tabular}{|c|c|c|}
\hline $\begin{array}{l}\text { PD Concentration } \\
(\mu M)\end{array}$ & Additions & $\begin{array}{c}\text { ATP Synthes is } \\
\text { (umoles } / \mathrm{hr} / \mathrm{mg} \text { chlorophyll) }\end{array}$ \\
\hline 0 & None & 2 \\
\hline$"$ & DBMIB $(10 \mu M)$ & $i$ \\
\hline$"$ & DCMU $(10 \mu M)^{\prime}$ & 0 \\
\hline$"$ & DCMU $(10 \mu \mathrm{M})+$ DBMIB $(10 \mu \mathrm{M})$ & 0 \\
\hline 62.5 & None & 106 \\
\hline " & DBMIB $(10 \mu \mathrm{M})$ & 25 \\
\hline " & DCMU $(10 \mu M)$ & 7 \\
\hline$"$ & DCMU $(10 \mu M)+$ DBMIB $(10 \mu M)$ & 0 \\
\hline 1300 & None & 196 \\
\hline " & DBMIB $(10 \mu \mathrm{M})$ & 108 \\
\hline$"$ & DCMU $(10 \mu M)$ & 68 \\
\hline & DCMU $(10 \mu M)+$ DBMIB $(10 \mu M)$ & 59 \\
\hline
\end{tabular}

* Assay conditions are given in "Materials and Methods." Illumination time was $1 \mathrm{~min}$.

ments with an oxygen electrode; under none of the conditions in Table II was oxygen uptake observed. This finding showed that the appropriate combination of PD $(62.5 \mu M)$ and DBMIB $(10 \mu M)$ created a condition under which photosystem II cyclic photophosphorylation occurs, by virtue of the fact that electron transfer between photosystem II and photosystem I is blocked by the DBMIB, and the photophosphorylation observed under these conditions is completely sensitive to DCMU.

If the ATP synthesis seen in $\mathrm{NH}_{2} \mathrm{OH}$-treated chloroplasts under the conditions just described was in fact due to a cyclic process, this reaction should be inhibited by conditions which would interrupt cyclic electron flow. Since PD was added in reduced form, addition of ferricyanide, which oxidizes PO, should affect ATP synthesis. The ferrocyanide formed by PD oxidation might also affect the cycle, since ferrocyanide has been shown to donate electrons to photosystem II in a reaction which does not support phosphorylation (11). Table III shows the effect of ferri-and ferrocyanide on PD-catalyzed photophosphorylation with $\mathrm{NH}_{2} \mathrm{OH}$-treated chloroplasts in the presence and absence of DBMIB. Ferricyanide inhibits ATP synthesis $77 \%$ in the absence of DBMIB and 
Table III

Effect of Ferri- and Ferrocyanide on PD-Catalyzed Photophosphorylation*

Additions

None

Ferricyanide $(125 \mu \mathrm{M})$

Ferrocyanide $(125 \mu \mathrm{M})$

DBMIB $(10 \mu \mathrm{M})$

DBMIB $(10 \mu M)+$ Ferricyanide $(125 \mu M)$

DBMIB $(10 \mu \mathrm{M})+$ Ferrocyanide $(125 \mu \mathrm{M})$
ATP Synthesis

(umoles/hr/mg chlorophyll)

*The concentration of P-PD was $62.5 \mu \mathrm{M}$ throughout. I1lumination time was 1 $\min$.

$100 \%$ in the presence of DBMIB. Ferrocyanide at equivalent concentrations has little effect in the absence of DBMIB. In the presence of DBMIB, however, a $65 \%$ inhibition of ATP synthes is is observed.

The nature of this inhibition was further explored with an $0_{2}$ electrode. In the presence of DBMIB, slow $\mathrm{O}_{2}$ uptake was observed $(5 \mu \mathrm{moles} / \mathrm{hr} / \mathrm{mg}$ chlorophyl1) under the conditions shown in Table III when either ferri- or ferrocyanide was added. It is therefore likely that the strong inhibitions of ATP synthesis seen in the presence of DBMIB when ferri- or ferrocyanide was present is due to oxidation of PD (by ferricyanide) as well as to photooxidation of ferrocyanide by photosystem II with transfer of electrons to $\mathrm{O}_{2}$ via DBMIB. This inhibition provides further evidence for the contention that the ATP synthesis obtained in the presence of DBMIB and PD is due to photosystem II cyclic photophosphorylation. DISCUSSION. The results in this communication provide evidence for a new pathway of ATP synthes is in isolated chloroplasts, namely photosystem II cyclic photophosphorylation. This cycle requires an artificial catalyst (PD) along with inhibitors (KCN, DBMIB) to block electron transport between the two photosystems and the ATP yield of the cycle is increased more than twofold by destruction of $\mathrm{O}_{2}$ evolution. A preliminary report has appeared, suggesting another PSII cycle which requires catechol and DBMIB (12). It is 
thus apparent that under the appropriate conditions, ATP-yielding reactions via cyclic electron flow are not unique to photosystem I. It is also apparent, from the conditions necessary to elicit the photosystem II cycle, that this reaction probably does not represent a naturally occurring path of ATP synthesis such as that seen with ferredoxin in photosystem I.

The experiments reported here cannot give a true assessment of the efficiency of ATP synthesis catalyzed by the cycle since concentrations of $P D$ in excess of $65 \mu M$ produce a reaction which is not completely inhibited by DBMIB plus DCMU. The requirement for DBMIB in $\mathrm{NH}_{2} \mathrm{OH}$-treated chloroplasts in fact presents an added complication. Since this compound is capable of oxidation-reduction (10), it is quite likely that it is an active participant in the cycle. It is probable, however, that oxygen is not reacting in the cycle. All attempts to demonstrate $0_{2}$ uptake under conditions favoring ATP synthesis were unsuccessful. Only in the presence of $125 \mu \mathrm{M}$ ferrocyanide pT us $10 \mu$ M DBMIB was any $\mathrm{O}_{2}$ uptake observed, and under these conditions ATP synthesis was more than $60 \%$ inhibited.

The discovery of conditions for the assay of photosystem II cyclic photophosphorylation is a consequence of the finding that there is a phosphorylation site associated with photosystem II. The fact that the catalyst for the cyclic reaction, PD, liberates protons as well as electrons upon oxidation supports the proposal set forth by Izawa and Ort (11) that a proton generating system, either water or an artificial donor, is required for coupling of ATP synthesis to photosystem II electron transport. The photosystem II cyclic reaction provides a convenient means for further studies on ATP synthesis associated with this light reaction in the absence of water oxidation. Experiments are currently under way to prepare chloroplasts treated with both $\mathrm{NH}_{2} \mathrm{OH}$ and $\mathrm{KCN}$ so that the photosystem II cycle may be assayed without interference from photosystem I-mediated reactions. Acknowledgements. The many helpful suggestions of Dr. H. Ikuma are gratefutly acknowledged. 


\section{REFERENCES}

1. Ouitrakul, R. and Izawa, S. (1973), Biochim. Biophys. Acta 305, 105-118.

2. Izawa, S., Gould, J.M., Ort, D.R., Felker, P., and Good, N.E. (1973), Biochim. Biophys. Acta 305, 119-128.

3. Saha, S., Ouitrakul, R., Tzawa, S., and Good, N.E. (1971), J. Bio1, Chem. 246, 3204-3209.

4. Ort, D.R., and Izawa, S. (1973), P1. Physiol, 53, 370-376.

5. Hauska, G., Reimer, S., and Trebst, A. (1974), Biochim. Biophys. Acta 357, 1-13.

6. Arnon, D.I. (1949), P1. Physiol. 24, 1-15.

7. Ort, D.R., and Izawa, S. (1973), PT. Physiol. 52, 595-600.

8. Avron, M. (1960), Biochim. Biophys. Acta 40, 257-272.

9. Izawa, S., Kraayenhof, R., Ruuge, E.K., and Devault, D. (1973), Biochim. Biophys.Acta 314, 328-339.

10. Gould, J.M. and Izawa, S. (1973), Eur. J. Biochem. 37, 185-192.

11. Izawa, S. and Ort, D.R. (1974), Biochim. Biophys. Acta 357, 127-143.

12. Izawa, S. (1975), P1. Physio1. 56(supp.), 55. 